\title{
A Simple Load Balancing Problem with Decentralized Information
}

\author{
Dimitrios G. Pandelis \\ Environmental Research Institute of Michigan, 3300 Plymouth Rd., Ann Arbor, MI 48105, USA \\ DeMOSTHENIS TENEKETZIS
}

Department of Electrical Engineering and Computer Science, University of Michigan, Ann Arbor, MI 48109-2122, USA

\begin{abstract}
The following load balancing problem is investigated in discrete time: A service system consists of two service stations and two controllers, one in front of each station. The service stations provide the same service with identical service time distributions and identical waiting costs. Customers requiring service arrive at a controller's site and are routed to one of the two stations by the controller. The processes describing the two arrival streams are identical. Each controller has perfect knowledge of the workload in its own station and receives information about the other station's workload with one unit of delay. The controllers' routing strategies that minimize the customers' total flowtime are determined for a certain range of the parameters that describe the arrival process and the service distribution. Specifically, we prove that optimal routing strategies are characterized by thresholds that are either precisely specified or take one of two possible values.
\end{abstract}

\section{Introduction}

We study optimal routing strategies for a queueing network consisting of two identical service stations with their associated queues and two identical arrival streams of jobs with associated controllers that have to route the jobs to one of the two service stations. Each controller can only observe the length of one queue. Information about the length of the other queue is received from the other controller, but it is subject to one time unit delay. We determine routing strategies that minimize the finite horizon expected total waiting time for a certain range of the parameters that describe the arrival and service processes.

This problem falls into the general context of decentralized control, where multiple decision makers, each one based on an imperfect knowledge of the state of the system, take actions to maximize a common performance measure. It is motivated by applications in communication and transportation networks consisting of several nodes that act individually, each one having a perfect knowledge of its local environment and an imperfect knowledge of the rest of the system. This imperfect knowledge may result either from lack of communication among the different nodes, or, when there is exchange of information, from propagation and processing delays. 
Optimal routing strategies with respect to various performance criteria for systems of parallel queues under centralized information have been studied extensively (see, for example, Davis (1977), Weber (1978), Ephremides et al. (1980), Bell and Stidham (1983), Hajek (1984), Lin and Kumar (1984), Stidham (1985), and Whitt (1986)). In the case of identical processors it is optimal to assign to each processor the same amount of work. When the queue lengths are available to the controller and service times are exponential it has been shown that the policy that assigns jobs to the shortest queue is optimal (see, for example, Winston (1977), Weber (1978), Ephremides et al. (1980), and Hordijk and Koole (1990)). A counterexample to the optimality of the "join-the-shortestqueue" policy when service times are not exponential is provided by Whitt (1986). When the queue lengths are not available to the controller and service times are exponential the Round-Robin policy that alternates among queues has been shown to be optimal (see Ephremides et al. (1980)). A randomized Round-Robin policy has also been shown to be optimal (see Stamoulis and Tsitsiklis (1991)) when the processors are identical, there are multiple identical arrival streams of customers and one controller associated with each stream, and the queue lengths are not available to any controller (i.e., all controllers have exactly the same information). In the case of nonidentical processors it has been shown that the optimal routing strategy is of the threshold type (see, for example, Hajek (1984), Lin and Kumar (1984), Walrand (1984), and Xu (1992) for the case of perfect information, and Beutler and Teneketzis (1989) for the case of imperfect information).

In contrast to problems with centralized information, work on routing problems with decentralized information has only concentrated on the performance evaluation of various strategies (see, for example, Boel and van Schuppen (1989), Hajek (1990), and Chang (1992)). In this paper we study optimal dynamic routing strategies for the simple load balancing problem with decentralized information presented at the beginning of this section. We prove that for a certain range of the parameters that describe the arrival process and the service distribution optimal routing strategies are characterized by thresholds. These thresholds are either precisely determined or take one of two possible values. Even though the model considered in this paper is quite restrictive, the determination of optimal routing strategies turns out to be a challenging task, and the analysis provides useful insights into the subtleties that arise in routing problems with decentralized information.

The paper is organized as follows. In Section 2 the formulation of a simple load balancing problem is presented. The problem is analyzed in Section 3, where an optimal routing strategy is explicitly determined for a certain range of the parameters that describe the arrival process and the service distribution. Conclusions appear in Section 4. 


\section{Problem Formulation}

We consider in discrete time the queueing system depicted in Figure 1. We have two controllers $C_{1}$ and $C_{2}$, and two queues $Q_{1}$ and $Q_{2}$. Jobs arrive to the two controllers in two independent arrival streams. The arrival streams are Bernoulli with parameter $\lambda$ (that is, at each time instant $t$ a job arrives with probability a and no jobs arrive with probability $1-\lambda$ ). Each controller has to decide whether to send a newly-arrived job to $Q_{1}$ or $Q_{2}$. Each queue is served by an independent server. Service times in both queues are geometric with parameter $\mu$. We make the following assumptions:

(A1) $\lambda<\mu$ for stability;

(A2) A job that arrives at a controller's site at time $t$ joins the queue to which it is routed at time $t+1$; and

(A3) At each time epoch departures occur after routing decisions.

Let $x_{t}^{i}, i=1,2$, be the length of $Q_{i}$ at time $t$ (including any job in service), $u_{t}^{i}$, $i=1,2$, the control action, if any, taken by $C_{i}$ at time $t$, and $z_{t}^{i}, i=1,2$, the information available to $C_{i}$ at time $t$. The control action $u_{t}^{i}$ is equal to 1 (respectively 2) when a job is routed to $Q_{1}$ (respectively $Q_{2}$ ). In the problem we are considering, controller $C_{1}$ (respectively $C_{2}$ ) knows the length of $Q_{1}$ (respectively $Q_{2}$ ) and has a one-step delay information about the length of $Q_{2}$ (respectively $Q_{1}$ ) and the control actions of $C_{2}$ (respectively $C_{1}$ ). Specifically

$$
\begin{aligned}
& z_{t}^{1}=\left(x_{s}^{1}, u_{s}^{1}, x_{s}^{2}, u_{s}^{2}, s=1,2, \ldots, t-1, x_{t}^{1}\right) \\
& z_{t}^{2}=\left(x_{s}^{1}, u_{s}^{1}, x_{s}^{2}, u_{s}^{2}, s=1,2, \ldots, t-1, x_{t}^{2}\right) .
\end{aligned}
$$

Our objective is to determine optimal routing strategies for the two controllers that minimize the expected total waiting time for all jobs that arrive strictly before time $T$, i.e.,

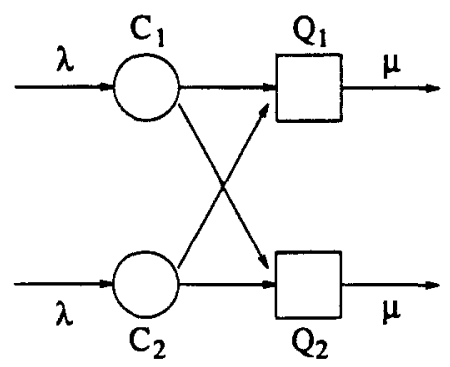

Fig. 1 


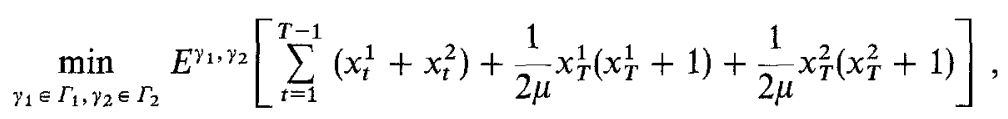

where $\Gamma_{i}, i=1,2$, is the set of all measurable functions of the data of controller $C_{i}$ taking values in $\{1,2\}$. Without loss of optimality (see Witsenhausen (1971), Varaiya and Walrand (1978)) we restrict attention to routing strategies of the form

$$
u_{t}^{i}=\gamma_{t}^{i}\left(x_{t}^{i}, \pi_{t}\right), \quad i=1,2
$$

where $x_{t}^{i}$ (the length of $Q_{i}$ at time $t$ ) is the private information of controller $C_{i}$ at $t$, and $\pi_{t}$ is the information common to both controllers at time $t$. The common information can be expressed as $\pi_{t}=\left(\pi_{t}^{1}, \pi_{t}^{2}\right)$, where $\pi_{t}^{i}, i=1,2$, denotes the probability distribution on the length of $Q_{i}$, based on information up to time $t-1$. We seek to determine optimal routing policies of the form (4).

\section{Analysis-Solution}

In this section we present the main ideas of the analysis and solution of the decentralized load balancing problem formulated in Section 2. All the details of the analysis can be found in Pandelis and Teneketzis (1993).

When it is common knowledge that the length of $Q_{i}$ at time $t-1$ is zero, then, because of assumptions (A2), (A3), and the specification of the information structure of the problem, it is common knowledge that the length of $Q_{i}$ at time $t$ is equal to some constant $x_{i}$ with probability 1 since there can be no departures from $Q_{i}$ at time $t-1$. We denote this situation by

$$
\pi_{t}^{i}=x_{i}
$$

Depending on the number of jobs routed to $Q_{i}$ at time $t-1, x_{i}$ can be equal to 0,1 , or 2 . When it is common knowledge that the length of $Q_{i}$ at time $t-1$ is nonzero, then, depending on the number of jobs routed to $Q_{i}$ at time $t-1$, it is common knowledge that the length of $Q_{i}$ at time $t$ is some $x_{i}$ with probability $1-\mu$ and $x_{i}-1$ with probability $\mu .{ }^{1}$ We denote this situation by

$$
\pi_{t}^{i}=\left(\begin{array}{c}
x_{i} \\
x_{i}-1
\end{array}\right)
$$

For example, if it is common knowledge that the length of $Q_{i}$ at $t-1$ is $\alpha_{i}>0$ and the number of jobs routed to $Q_{i}$ at $t-1$ is $\beta_{i}$, then $x_{i}=\alpha_{i}+\beta_{i}$. 
Let $V_{t}\left(\pi_{t}^{1}, \pi_{t}^{2}\right)$ be the minimum expected total waiting time incurred from time $t$ on for all jobs when the common information at time $t$ is $\left(\pi_{t}^{1}, \pi_{t}^{2}\right)$. The optimality condition for $V_{t}\left(\pi_{t}^{1}, \pi_{t}^{2}\right)$ is (see Varaiya and Walrand (1978))

$$
\begin{array}{r}
V_{t}\left(\pi_{t}^{1}, \pi_{t}^{2}\right)=E^{\pi_{t}^{1}}\left(x_{t}^{1}\right)+E^{\pi_{t}^{2}}\left(x_{t}^{2}\right)+\min _{\left(\gamma_{t}^{1}, \gamma_{t}^{2}\right)} W_{t+1}\left(\pi_{t}^{1}, \pi_{t}^{2}, \gamma_{t}^{1}, \gamma_{t}^{2}\right), \\
t=1,2, \ldots, T-1, \\
V_{T}\left(\pi_{T}^{1}, \pi_{T}^{2}\right)=E^{\pi_{T}^{1}}\left[\frac{1}{2 \mu} x_{T}^{1}\left(x_{T}^{1}+1\right)\right]+E^{\pi_{T}^{2}}\left[\frac{1}{2 \mu} x_{T}^{2}\left(x_{T}^{2}+1\right)\right],
\end{array}
$$

where $W_{t+1}\left(\pi_{t}^{1}, \pi_{t}^{2}, \gamma_{t}^{1}, \gamma_{t}^{2}\right)$ is the minimum expected total waiting time for all jobs from time $t+1$ on when the common information at time $t$ is $\left(\pi_{t}^{1}, \pi_{t}^{2}\right)$ and the control law at time $t$ is $\left(\gamma_{t}^{1}, \gamma_{t}^{2}\right)$.

To determine optimal routing strategies of controllers $C_{1}$ and $C_{2}$ we need to specify their optimal actions for each possible value of the information state $\left(\pi_{t}^{1}, \pi_{t}^{2}\right)$. We proceed in the following steps:

Step 1: For every and each state $\left(\pi_{t}^{1}, \pi_{t}^{2}\right)$ we explicitly specify all the possible routing strategies $\left(\gamma_{t}^{1}, \gamma_{t}^{2}\right)$ and determine analytically the form of $W_{t+1}\left(\pi_{t}^{1}, \pi_{t}^{2}, \gamma_{t}^{1}, \gamma_{t}^{2}\right)$.

Step 2: We conjecture properties of the minimum expected total waiting time function $V_{t}\left(\pi_{t}^{1}, \pi_{t}^{2}\right)$ and using these properties we determine an optimal routing strategy $\left(\gamma_{t}^{1}, \gamma_{t}^{2}\right)$ for each $t$.

Step 3: We prove inductively the conjectured properties of the function $V_{t}\left(\pi_{t}^{1}, \pi_{t}^{2}\right)$ using the optimal routing strategy found in Step 2 .

The properties of $V_{t}\left(\pi_{t}^{1}, \pi_{t}^{2}\right)$ and an optimal routing strategy are presented in Theorems 1 and 2 that follow. The details of the analysis of each step and the proofs of Theorems 1 and 2 are given in Pandelis and Teneketzis (1993). Note that we have only been able to prove Theorems 1 and 2 for the following regions of the parameters $\lambda$ and $\mu$ :

$$
\begin{aligned}
& \frac{1}{2} \leq \lambda<\mu, \\
& \lambda<\mu \leq \frac{1}{2} .
\end{aligned}
$$

When $\lambda<\frac{1}{2}<\mu$ we show by example that the optimal routing strategies explicitly depend on the specific values of $\lambda$ and $\mu$. For this reason we have been 
unable to derive any properties of optimal routing strategies in this case. We proceed by stating the properties of the minimum expected total waiting time function $V_{t}\left(\dot{\pi}_{t}^{1}, \pi_{t}^{2}\right)$ in the following theorem.

Theorem 1: For $\frac{1}{2} \leq \lambda<\mu$ and $\lambda<\mu \leq \frac{1}{2}$ the minimum expected total waiting time function $V_{t}\left(\pi_{t}^{1}, \pi_{t}^{2}\right)$ satisfies the following properties:

(P1): For any $\pi^{1}, \pi^{2}$ and for all $t$ we have

$$
V_{t}\left(\pi^{1}, \pi^{2}\right)=V_{t}\left(\pi^{2}, \pi^{1}\right)
$$

(P2): If $\pi^{1}, \pi^{2}, \tilde{\pi}^{1}, \tilde{\pi}^{2}$ are such that

$$
\begin{aligned}
& E^{\pi^{1}}\left(x_{t}^{1}\right)+E^{\pi^{2}}\left(x_{t}^{2}\right)=E^{\tilde{\pi}^{1}}\left(x_{t}^{1}\right)+E^{\tilde{\pi}^{2}}\left(x_{t}^{2}\right), \quad \text { and } \\
& \pi^{1} \underset{\text { st }}{<} \tilde{\pi}^{1}, \tilde{\pi}^{2}{\underset{\mathrm{st}}{\mathrm{t}}}^{2}, \quad \text { or } \\
& \pi^{2} \pi_{\mathrm{st}} \tilde{\pi}^{1}, \tilde{\pi}^{2}{\underset{\mathrm{st}}{\mathrm{t}}}^{1}, \quad \text { then } \\
& V_{t}\left(\pi^{1}, \pi^{2}\right) \geq V_{t}\left(\tilde{\pi}^{1}, \tilde{\pi}^{2}\right), \quad t=1,2, \ldots, T .^{2}
\end{aligned}
$$

(P3): If $\pi^{1} \underset{\mathrm{st}}{\leq} \tilde{\pi}^{1}$ and $\pi^{2} \underset{\mathrm{st}}{\leq} \tilde{\pi}^{2}$, then

$$
V_{t}\left(\pi^{1}, \pi^{2}\right) \leq V_{t}\left(\tilde{\pi}^{1}, \tilde{\pi}^{2}\right), \quad t=1,2, \ldots, T
$$

(P4): For $\frac{1}{2} \leq \lambda<\mu$ we have

$$
V_{t}\left(2, \begin{array}{l}
1 \\
0
\end{array}\right)-V_{t}\left(1, \begin{array}{l}
2 \\
1
\end{array}\right) \geq V_{t}\left(0, \begin{array}{l}
2 \\
1
\end{array}\right)-V_{t}\left(1, \begin{array}{l}
1 \\
0
\end{array}\right), \quad t=1,2, \ldots, T
$$

For $\lambda<\mu \leq \frac{1}{2}$ we have

$$
V_{t}\left(2, \begin{array}{l}
1 \\
0
\end{array}\right)-V_{t}\left(1, \begin{array}{l}
2 \\
1
\end{array}\right) \leq V_{t}\left(0, \begin{array}{l}
2 \\
1
\end{array}\right)-V_{t}\left(1, \begin{array}{l}
1 \\
0
\end{array}\right), \quad t=1,2, \ldots, T
$$

$2 \pi_{s i}^{i} \tilde{\pi}^{j}$ means that a random variable $X^{i}$ with probability distribution $\pi^{i}$ is stochastically smaller than a random variable $\tilde{X}^{j}$ with probability distribution $\tilde{\pi}^{j}\left(\sec\right.$ Ross (1983)) and $\pi^{i}, \tilde{\pi}^{j}$ are not identical. For the case when $\pi^{i}, \tilde{\pi}^{j}$ can be identical we use the notation $\pi^{i} \frac{\leq}{\mathrm{st}} \tilde{\pi}^{j}$. 
We now interpret these properties. Since (i) the two queues have probabilistically the same service time and the same waiting cost, and (ii) the arrival processes at the two controllers are probabilistically the same and the two controllers have symmetric information, the optimal performance remains the same when the common beliefs about the queue lengths are interchanged. This is illustrated by property (P1). Property (P2) expresses the fact that the performance is improved when the load is balanced between the two queues. One would expect that if the number of jobs originally present in each queue is stochastically increased, then the minimum expected total waiting time would increase. This is expressed by property (P3). Property (P4) is more subtle and related to the load balancing idea. It says the following: Consider the load of the queues expressed by $\left(\pi^{1}, \pi^{2}\right)$ before a newly-arrived job is assigned to one of the two queues. Then, as the load before the assignment becomes more "unbalanced," the difference in performance resulting by the two possible assignments of the newly-arrived job increases.

Based on these properties we prove for regions (R1) and (R2) that there exists an optimal routing strategy that is characterized by thresholds. ${ }^{3}$ We show that for each $\left(x_{t}^{1}, \pi_{t}^{1}\right)\left(\left(x_{t}^{2}, \pi_{t}^{2}\right)\right)$ there exists a distribution $\ell_{t}^{1, *}\left(x_{t}^{1}, \pi_{t}^{1}\right)\left(\ell_{t}^{2, *}\left(x_{t}^{2}, \pi_{t}^{2}\right)\right)$ such that an optimal routing strategy, denoted by $\left(\gamma_{t}^{1, *}, \gamma_{t}^{2, *}\right), t=1,2, \ldots, T$, is given by

$$
\begin{array}{lll}
\gamma_{t}^{1, *}\left(x_{t}^{1}, \pi_{t}^{1}, \pi_{t}^{2}\right)=1 & \text { for } \pi_{t}^{2} \underset{\mathrm{st}}{\gtrless} \ell_{t}^{1, *}\left(x_{t}^{1}, \pi_{t}^{1}\right), & \text { and } \\
\gamma_{t}^{1, *}\left(x_{t}^{1}, \pi_{t}^{1}, \pi_{t}^{2}\right)=2 & \text { for } \pi_{t}^{2} \underset{\mathrm{st}}{\widehat{t}} \ell_{t}^{1, *}\left(x_{t}^{1}, \pi_{t}^{1}\right) \\
\left(\gamma_{t}^{2, *}\left(x_{t}^{2}, \pi_{t}^{1}, \pi_{t}^{2}\right)=2\right. & \text { for } \pi_{t}^{1} \underset{\mathrm{st}}{\gtrless} \ell_{t}^{2, *}\left(x_{t}^{2}, \pi_{t}^{2}\right), & \text { and } \\
\gamma_{t}^{2, *}\left(x_{t}^{2}, \pi_{t}^{1}, \pi_{t}^{2}\right)=1 & \text { for } \left.\pi_{t}^{1} \underset{\mathrm{st}}{\gtrless} \ell_{t}^{2, *}\left(x_{t}^{2}, \pi_{t}^{2}\right)\right) . &
\end{array}
$$

Furthermore, we show that each $\ell_{t}^{1, *}\left(x_{t}^{1}, \pi_{t}^{1}\right)\left(\ell_{t}^{2, *}\left(x_{t}^{2}, \pi_{t}^{2}\right)\right)$ is either precisely specified or it is one of two possible distributions, by explicitly determining the optimal routing decisions for all states except a subset where we reduce the search from 16 to 2 possible decisions. We proceed with the analysis of regions (R1) and (R2).

First we note that it is straightforward to show that the optimal routing strategy is symmetric. This symmetry is expressed by the relation

$$
\gamma_{t}^{1, *}(x, \pi, \tilde{\pi}) \neq \gamma_{t}^{2, *}(x, \tilde{\pi}, \pi), \quad \text { for any } x, \pi, \tilde{\pi}
$$

3 It is easy to show that there are more than one optimal routing strategies. All optimal strategies determined by our approach are characterized by the rule "keep-the-queue-lengths-as-balanced-as possible." However, not all optimal strategies are characterized by thresholds. 
That is, if based on the information $(x, \pi, \tilde{\pi}) C_{1}$ routes an incoming job to $Q_{1}$ (respectively $Q_{2}$ ), then based on $(x, \tilde{\pi}, \pi) C_{2}$ routes an incoming job to $Q_{2}$ (respectively $Q_{1}$ ). In terms of the optimal thresholds the symmetry is expressed by $\ell_{t}^{1, *}(x, \pi)=\ell_{t}^{2, *}(x, \pi)$, for any $x, \pi$.

Now we analyze regions (R1) and (R2) separately.

Case $1: \frac{1}{2} \leq \lambda<\mu$.

An optimal routing strategy is the following:

$$
\gamma_{t}^{1, *}\left(\begin{array}{ccc}
1 & 1 & 1 \\
1 & 0 & 0
\end{array}\right)=1, \quad \gamma_{t}^{1, *}\left(\begin{array}{lll}
0, & 1 & 1 \\
& 0 & 0
\end{array}\right)=1
$$

For $x \geq 2$,

$$
\begin{aligned}
& \gamma_{t}^{1 * *}\left(x, \begin{array}{cc}
x & x \\
x-1 & x-1
\end{array}\right)=2, \quad \gamma_{t}^{1, *}\left(x-1, \begin{array}{cc}
x & x \\
x-1^{\prime} x-1
\end{array}\right)=1 . \\
& \gamma_{t}^{1, *}\left(1, \begin{array}{cc}
1 & 2 \\
0 & 0
\end{array}\right)=1, \quad \gamma_{t}^{1, *}\left(\begin{array}{lll}
0, & 1 & 2 \\
0 & 1 & 1
\end{array}\right)=1 \\
& \gamma_{t}^{2, *}\left(\begin{array}{ccc}
2, & 1 & 2 \\
0 & 0
\end{array}\right)=1, \quad \gamma_{t}^{2, *}\left(\begin{array}{ccc}
1, & 1 & 2 \\
0 & 0 & 1
\end{array}\right)=2
\end{aligned}
$$

For $x \geq 2$,

$$
\begin{aligned}
& \gamma_{t}^{1 * *}\left(x, \begin{array}{cc}
x & x+1 \\
x-1 & x
\end{array}\right)=2, \quad \gamma_{t}^{1 * *}\left(\begin{array}{cc}
x-1, & x, 1, \\
x-1 & x+1
\end{array}\right)=1, \\
& \gamma_{t}^{2 * *}\left(x+1, \begin{array}{c}
x-x+1 \\
x-1
\end{array}\right)=1, \quad \gamma_{t}^{2, *}\left(\begin{array}{cc}
x, & x+1 \\
x-1 & x+1 \\
x
\end{array}\right)=1 \text {, }
\end{aligned}
$$

or

$$
\begin{aligned}
& \gamma_{t}^{1, *}\left(\begin{array}{cc}
x, & x+1 \\
x-1 & x
\end{array}\right)=1, \quad \gamma_{t}^{1 * *}\left(x-1, \begin{array}{c}
x, \\
x-1, \\
x+1
\end{array}\right)=1, \\
& \gamma_{t}^{2 * *}\left(x+1, \begin{array}{cc}
x & x+1 \\
x-1 & x
\end{array}\right)=1, \quad \gamma_{t}^{2, *}\left(\begin{array}{cc}
x, & x+1 \\
x-1 & x+1 \\
x
\end{array}\right)=2 .
\end{aligned}
$$


For $x \geq 1, y \geq x+2$,

$$
\begin{aligned}
& \gamma_{t}^{1, *}\left(\begin{array}{c}
x, y \\
x-1, y-1
\end{array}\right)=1, \quad \gamma_{t}^{1, *}\left(x-1, \begin{array}{cc}
x & y \\
x-1 & y-1
\end{array}\right)=1, \\
& \gamma_{t}^{2, *}\left(\begin{array}{c}
x, y \\
x-1, y-1
\end{array}\right)=1, \quad \gamma_{t}^{2, *}\left(y-1, \begin{array}{c}
x \\
x-1, y-1
\end{array}\right)=1 . \\
& \gamma_{t}^{1, *}\left(0,0, \begin{array}{c}
1 \\
0
\end{array}\right)=1, \quad \gamma_{t}^{2, *}\left(1,0, \begin{array}{l}
1 \\
0
\end{array}\right)=2, \quad \gamma_{t}^{2, *}\left(0,0, \begin{array}{l}
1 \\
0
\end{array}\right)=2 . \\
& \gamma_{t}^{1, *}\left(0,0, \begin{array}{l}
2 \\
1
\end{array}\right)=1, \quad \gamma_{t}^{2, *}\left(2,0, \begin{array}{l}
2 \\
1
\end{array}\right)=1, \quad \gamma_{t}^{2, *}\left(1,0, \begin{array}{l}
2 \\
1
\end{array}\right)=2 .
\end{aligned}
$$

For $x \geq 3$,

$$
\begin{aligned}
& \gamma_{t}^{1, *}\left(0,0, \begin{array}{c}
x \\
x-1
\end{array}\right)=1, \quad \gamma_{t}^{2, *}\left(x, 0, \begin{array}{c}
x \\
x-1
\end{array}\right)=1, \\
& \gamma_{t}^{2, *}\left(x-1,0, \begin{array}{c}
x \\
x-1
\end{array}\right)=1 . \\
& \gamma_{t}^{1, *}\left(1,1, \frac{1}{0}\right)=1, \quad \gamma_{t}^{2, *}\left(1,1, \begin{array}{l}
1 \\
0
\end{array}\right)=2, \quad \gamma_{t}^{2, *}\left(0,1, \frac{1}{0}\right)=2 . \\
& \gamma_{t}^{1, *}\left(2,2, \begin{array}{l}
1 \\
0
\end{array}\right)=2, \quad \gamma_{t}^{2, *}\left(1,2, \begin{array}{l}
1 \\
0
\end{array}\right)=2, \quad \gamma_{t}^{2, *}\left(0,2, \begin{array}{l}
1 \\
0
\end{array}\right)=2 . \\
& \gamma_{t}^{1, *}\left(2,2, \begin{array}{l}
2 \\
1
\end{array}\right)=2, \quad \gamma_{t}^{2, *}\left(2,2, \frac{2}{1}\right)=1, \quad \gamma_{t}^{2, *}\left(1,2, \begin{array}{l}
2 \\
1
\end{array}\right)=2 .
\end{aligned}
$$

For $x=1$ or 2 ,

$$
\begin{aligned}
& \gamma_{t}^{1, *}\left(x, x, \begin{array}{c}
x+1 \\
x
\end{array}\right)=1, \quad \gamma_{t}^{2, *}\left(x+1, x, \begin{array}{c}
x+1 \\
x
\end{array}\right)=1, \\
& \gamma_{t}^{2, *}\left(x, x, \begin{array}{c}
x+1 \\
x
\end{array}\right)=2 .
\end{aligned}
$$


For $x=1$ or $2, y \geq x+2$,

$$
\begin{aligned}
& \gamma_{t}^{1, *}\left(x, x, \begin{array}{c}
y \\
y-1
\end{array}\right)=1, \quad \gamma_{t}^{2, *}\left(y, x, \begin{array}{c}
y \\
y-1
\end{array}\right)=1 \\
& \gamma_{t}^{2, *}\left(y-1, x, \begin{array}{c}
y \\
y-1
\end{array}\right)=1 . \\
& \gamma_{t}^{1, *}(0,0,1)=1, \quad \gamma_{t}^{2, *}(1,0,1)=2 . \\
& \gamma_{t}^{1, *}(0,0,2)=1, \quad \gamma_{t}^{2, *}(2,0,2)=1 .
\end{aligned}
$$

For $x=0$ or 1 ,

$$
\gamma_{t}^{1, *}(x, x, x)=1, \quad \gamma_{t}^{2, *}(x, x, x)=2
$$

We conjecture that for $x \geq 2$ the control law given in (12) is optimal. To prove our conjecture it suffices to show that the value function $V_{t}\left(\pi_{t}^{1}, \pi_{t}^{2}\right)$ satisfies the following property:

$$
\begin{aligned}
& V_{t}\left(\begin{array}{cc}
x-1 & x+1 \\
x-2 & x
\end{array}\right)-V_{t}\left(\begin{array}{cc}
x & x \\
x-1 & x-1
\end{array}\right) \\
& \geq V_{t}\left(\begin{array}{cr}
x & x+2 \\
x-1 & x+1
\end{array}\right)-V_{t}\left(\begin{array}{cc}
x+1 & x+1 \\
x & x
\end{array}\right), \quad x \geq 2 .
\end{aligned}
$$

Intuitively (26) can be interpreted as follows: The left-hand side of (26) represents the incentive to route a job to $Q_{1}$ when the common information of the two controllers is $\left(\begin{array}{cc}x-1 & x \\ x-2 & x-1\end{array}\right)$. This incentive decreases when the common information changes from $\left(\begin{array}{cc}x-1, & x \\ x-2 & x-1\end{array}\right)$ to $\left(\begin{array}{cc}x, & x+1 \\ x-1 & x\end{array}\right)$. That is, the importance of making the "right routing decision" decreases as the length of the queues increases uniformly. We have been unable to prove (26).

Case 2: $\lambda<\mu \leq \frac{1}{2}$.

An optimal routing strategy is the following:

For $x \geq 1$,

$$
\gamma_{t}^{1, *}\left(\begin{array}{cc}
x, & x \\
x-1 & x-1
\end{array}\right)=2, \quad y_{t}^{1, *}\left(x-1, \begin{array}{cc}
x & x \\
x-1 & x-1
\end{array}\right)=1
$$


For $x \geq 1$,

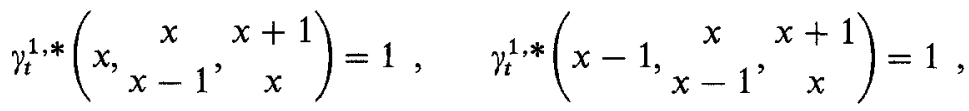

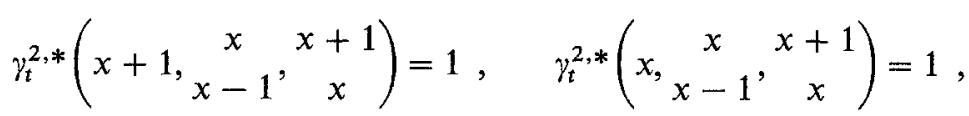

or

$$
\begin{aligned}
& \gamma_{t}^{1, *}\left(\begin{array}{cc}
x, & x+1 \\
x-1, & x
\end{array}\right)=1, \quad \gamma_{t}^{1, *}\left(x-1, \begin{array}{c}
x, \\
x-1, \\
x+1
\end{array}\right)=1
\end{aligned}
$$

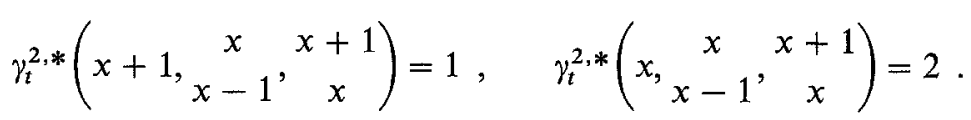

For $x \geq 1, y \geq x+2$,

$$
\begin{aligned}
& \gamma_{t}^{1, *}\left(\begin{array}{cc}
x, & y \\
x-1 & y-1
\end{array}\right)=1, \quad \gamma_{t}^{1, *}\left(x-1, \begin{array}{c}
x \\
x-1, y-1
\end{array}\right)=1, \\
& \gamma_{t}^{2, *}\left(\begin{array}{cc}
x & y \\
x-1, y-1
\end{array}\right)=1, \quad \gamma_{t}^{2, *}\left(y-1, \begin{array}{cc}
x & y \\
x-1, y-1
\end{array}\right)=1 . \\
& \gamma_{t}^{1, *}\left(2,2, \begin{array}{c}
1 \\
0
\end{array}\right)=2, \quad \gamma_{t}^{2, *}\left(1,2, \begin{array}{c}
1 \\
0
\end{array}\right)=2, \quad \gamma_{t}^{2, *}\left(\begin{array}{l}
0,2, \\
0
\end{array}\right)=2 .
\end{aligned}
$$

For $x=0,1$, or 2 ,

$$
\begin{aligned}
& \gamma_{t}^{1, *}\left(x, x, \begin{array}{c}
x+1 \\
x
\end{array}\right)=1, \quad \gamma_{t}^{2, *}\left(x+1, x, \begin{array}{c}
x+1 \\
x
\end{array}\right)=1, \\
& \gamma_{t}^{2, *}\left(x, x, \begin{array}{c}
x+1 \\
x
\end{array}\right)=2 .
\end{aligned}
$$

For $x=1$ or 2 ,

$$
\begin{aligned}
& \gamma_{t}^{1, *}\left(x, x, \begin{array}{c}
x \\
x-1
\end{array}\right)=2, \quad \gamma_{t}^{2, *}\left(x, x, \begin{array}{c}
x \\
x-1
\end{array}\right)=1 \\
& \gamma_{t}^{2, *}\left(x-1, x, \begin{array}{c}
x \\
x-1
\end{array}\right)=2 \text {. }
\end{aligned}
$$


For $x=0,1$ or $2, y \geq x+2$,

$$
\begin{aligned}
& \gamma_{t}^{1, *}\left(x, x, \begin{array}{c}
y \\
y-1
\end{array}\right)=1, \quad \gamma_{t}^{2, *}\left(y, x, \begin{array}{c}
y \\
y-1
\end{array}\right)=1, \\
& \gamma_{t}^{2, *}\left(y-1, x, \begin{array}{c}
y \\
y-1
\end{array}\right)=1 .
\end{aligned}
$$

For $x=1$ or 2 ,

$$
\gamma_{t}^{1, *}(0,0, x)=1, \quad \gamma_{t}^{2, *}(x, 0, x)=1
$$

For $x=0$ or 1 ,

$$
\gamma_{t}^{1, *}(x, x, x)=2, \quad \gamma_{t}^{2, *}(x, x, x)=1
$$

We conjecture that for $x \geq 2$ the strategy given by (28) is optimal. To prove the conjecture it would suffice to show that (26) is true.

The optimal routing strategies for regions (R1) and (R2) are summarized in the following theorem.

Theorem 2: For each $\left(x_{t}^{1}, \pi_{t}^{1}\right)\left(\left(x_{t}^{2}, \pi_{t}^{2}\right)\right)$ there exists a threshold distribution $\ell_{t}^{1, *}\left(x_{t}^{1}, \pi_{t}^{1}\right)\left(\ell_{t}^{2, *}\left(x_{t}^{2}, \pi_{t}^{2}\right)\right)$, where $\ell_{t}^{1, *}(x, \pi)=\ell_{t}^{2, *}(x, \pi)$ for any $x, \pi$, such that an optimal routing strategy is given by

$$
\begin{aligned}
& \gamma_{t}^{1, *}\left(x_{t}^{1}, \pi_{t}^{1}, \pi_{t}^{2}\right)=1 \quad \text { for } \quad \pi_{t}^{2} \underset{\mathrm{st}}{\gtrless} \ell_{t}^{1, *}\left(x_{t}^{1}, \pi_{t}^{1}\right), \quad \text { and } \\
& \gamma_{t}^{1, *}\left(x_{t}^{1}, \pi_{t}^{1}, \pi_{t}^{2}\right)=2 \quad \text { for } \quad \pi_{t}^{2}<_{\mathrm{st}} \ell_{t}^{1, *}\left(x_{t}^{1}, \pi_{t}^{1}\right) \\
& \left(\gamma_{t}^{2, *}\left(x_{t}^{2}, \pi_{t}^{1}, \pi_{t}^{2}\right)=2 \quad \text { for } \quad \pi_{t}^{1} \underset{\mathrm{st}}{\geq} \ell_{t}^{2, *}\left(x_{t}^{2}, \pi_{t}^{2}\right), \quad\right. \text { and } \\
& \left.\gamma_{t}^{2, *}\left(x_{t}^{2}, \pi_{t}^{1}, \pi_{t}^{2}\right)=1 \quad \text { for } \quad \pi_{t}^{1}<_{\mathrm{st}} \ell_{t}^{2, *}\left(x_{t}^{2}, \pi_{t}^{2}\right)\right) .
\end{aligned}
$$

The optimal thresholds are the following:

Case $1 . \frac{1}{2} \leq \lambda<\mu$.

$$
\ell_{t}^{1, *}\left(1, \begin{array}{l}
1 \\
0
\end{array}\right)=0
$$


A Simple Load Balancing Problem with Decentralized Information

109

$$
\begin{aligned}
& \ell_{t}^{1, *}\left(\begin{array}{c}
x, \\
x-1
\end{array}\right)=\left(\begin{array}{c}
x+1 \\
x
\end{array}\right) \text { or }\left(\begin{array}{l}
x+2 \\
x+1
\end{array}\right), \quad x \geq 2, \\
& \text { (we conjecture } \left.\left(\begin{array}{l}
x+2 \\
x+1
\end{array}\right)\right) \text {, } \\
& \ell_{t}^{1, *}\left(x-1, \begin{array}{c}
x \\
x-1
\end{array}\right)=0, \quad x=1,2 \text {, } \\
& \left.\ell_{t}^{1, *}\left(2, \begin{array}{l}
3 \\
2
\end{array}\right)=\left(\begin{array}{l}
2 \\
1
\end{array}\right) \text { or } 2 \text { (we conjecture } 2\right) \text {, } \\
& \ell_{t}^{1, *}\left(x-1, \begin{array}{c}
x \\
x-1
\end{array}\right)=\left(\begin{array}{l}
x-1 \\
x-2
\end{array}\right) \text { or }\left(\begin{array}{c}
x \\
x-1
\end{array}\right), \quad x \geq 4 \text {, } \\
& \left(\text { we conjecture }\left(\begin{array}{c}
x \\
x-1
\end{array}\right)\right) \text {, } \\
& \ell_{t}^{1, *}(x, x)=0, \quad x=0,1 \text {, } \\
& \ell_{t}^{1, *}(2,2)=\left(\begin{array}{l}
3 \\
2
\end{array}\right)
\end{aligned}
$$

Case 2. $\lambda<\mu \leq \frac{1}{2}$.

$$
\begin{aligned}
& \ell_{t}^{1, *}\left(\begin{array}{c}
x, \\
x-1
\end{array}\right)=\left(\begin{array}{c}
x+1 \\
x
\end{array}\right), \quad x \geq 1 \\
& \ell_{t}^{1, *}\left(\begin{array}{l}
1 \\
0, \\
0
\end{array}\right)=0 \\
& \ell_{t}^{1, *}\left(x-1, \begin{array}{c}
x \\
x-1
\end{array}\right)=\left(\begin{array}{l}
x-1 \\
x-2
\end{array}\right) \text { or } x-1, \quad x=2,3 \\
& \left(\text { we conjecture }\left(\begin{array}{l}
x-1 \\
x-2
\end{array}\right)\right) \text {, } \\
& \ell_{t}^{1, *}\left(x-1, \begin{array}{c}
x \\
x-1
\end{array}\right)=\left(\begin{array}{l}
x-1 \\
x-2
\end{array}\right) \text { or }\left(\begin{array}{c}
x \\
x-1
\end{array}\right), \quad x \geq 4 \text {, } \\
& \text { (we conjecture } \left.\left(\begin{array}{l}
x-1 \\
x-2
\end{array}\right)\right) \text {, } \\
& \ell_{t}^{1, *}(x, x)=\left(\begin{array}{c}
x+1 \\
x
\end{array}\right), \quad x=0,1,2 \text {. }
\end{aligned}
$$


When $\lambda<\frac{1}{2}<\mu$ the optimal routing strategy explicitly depends on the values of $\lambda$ and $\mu$. We illustrate this point by the following example.

Example 1. Let $\pi_{T-1}=\left(\pi_{T-1}^{1}, \pi_{T-1}^{2}\right)=(0,1)$ be the common information of the two controllers at time $T-1$. As defined before, the optimal control law $\left(\gamma_{T-1}^{1, *}, \gamma_{T-1}^{2, *}\right)$ is the one that minimizes $W_{T}\left(0,1, \gamma_{T-1}^{1}, \gamma_{T-1}^{2}\right)$. We have 4 possible control laws.

Control Law 1: $\gamma_{T-1}^{1}(0,0,1)=1, \gamma_{T-1}^{2}(1,0,1)=1$.

$$
\begin{aligned}
W_{T}\left(0,1, \gamma_{T-1}^{1}, \gamma_{T-1}^{2}\right)= & \lambda^{2} V_{T}\left(2, \begin{array}{l}
1 \\
0
\end{array}\right)+\lambda(1-\lambda) V_{T}\left(1, \begin{array}{l}
1 \\
0
\end{array}\right) \\
& +(1-\lambda) \lambda V_{T}\left(1, \begin{array}{l}
1 \\
0
\end{array}\right)+(1-\lambda)^{2} V_{T}\left(\begin{array}{l}
1 \\
0,
\end{array}\right)
\end{aligned}
$$

Control Law 2: $\gamma_{T-1}^{1}(0,0,1)=1, \gamma_{T-1}^{2}(1,0,1)=2$.

$$
\begin{aligned}
W_{T}\left(0,1, \gamma_{T-1}^{1}, \gamma_{T-1}^{2}\right)= & \lambda^{2} V_{T}\left(1, \begin{array}{l}
2 \\
1
\end{array}\right)+\lambda(1-\lambda) V_{T}\left(1, \begin{array}{l}
1 \\
0
\end{array}\right) \\
& +(1-\lambda) \lambda V_{T}\left(0, \begin{array}{l}
2 \\
1
\end{array}\right)+(1-\lambda)^{2} V_{T}\left(\begin{array}{l}
1 \\
0
\end{array}\right)
\end{aligned}
$$

Control Law 3: $\gamma_{T-1}^{1}(0,0,1)=2, \gamma_{T-1}^{2}(1,0,1)=1$.

$$
\begin{aligned}
W_{T}\left(0,1, \gamma_{T-1}^{1}, \gamma_{T-1}^{2}\right)= & \lambda^{2} V_{T}\left(1, \begin{array}{l}
2 \\
1
\end{array}\right)+\lambda(1-\lambda) V_{T}\left(0, \begin{array}{l}
2 \\
1
\end{array}\right) \\
& +(1-\lambda) \lambda V_{T}\left(1, \begin{array}{l}
1 \\
0
\end{array}\right)+(1-\lambda)^{2} V_{T}\left(\begin{array}{l}
1 \\
0,
\end{array}\right)
\end{aligned}
$$

Control Law 4: $\gamma_{T-1}^{1}(0,0,1)=2, \gamma_{T-1}^{2}(1,0,1)=2$.

$$
\begin{aligned}
W_{T}\left(0,1, \gamma_{T-1}^{1}, \gamma_{T-1}^{2}\right)= & \lambda^{2} V_{T}\left(0, \begin{array}{l}
3 \\
2
\end{array}\right)+\lambda(1-\lambda) V_{T}\left(0, \begin{array}{l}
2 \\
1
\end{array}\right) \\
& +(1-\lambda) \lambda V_{T}\left(0, \begin{array}{l}
2 \\
1
\end{array}\right)+(1-\lambda)^{2} V_{T}\left(\begin{array}{l}
1 \\
0
\end{array}\right)
\end{aligned}
$$


Using (8) we get

$$
\begin{aligned}
& V_{T}\left(2, \begin{array}{l}
1 \\
0
\end{array}\right)=\frac{4-\mu}{\mu}, \\
& V_{T}\left(1, \begin{array}{l}
2 \\
1
\end{array}\right)=\frac{4-2 \mu}{\mu}, \\
& V_{T}\left(1, \begin{array}{l}
1 \\
0
\end{array}\right)=\frac{2-\mu}{\mu}, \\
& V_{T}\left(\begin{array}{l}
2 \\
1
\end{array}\right)=\frac{3-2 \mu}{\mu}, \\
& V_{T}\left(\begin{array}{l}
3 \\
2
\end{array}\right)=\frac{6-3 \mu}{\mu} .
\end{aligned}
$$

Let $W_{T}^{1}, W_{T}^{2}, W_{T}^{3}$, and $W_{T}^{4}$ denote the expressions given in the right-hand sides of (37), (38), (39), and (40) respectively. First note that

$$
W_{T}^{2}=W_{T}^{3}
$$

From (41)-(45) we get

$$
\begin{aligned}
& W_{T}^{1}-W_{T}^{2}=\frac{\lambda}{\mu}(\lambda+\mu-1), \\
& W_{T}^{1}-W_{T}^{4}=\frac{\lambda}{\mu} 2(\mu-1)<0, \\
& W_{T}^{2}-W_{T}^{4}=\frac{\lambda}{\mu} 2(\mu-\lambda-1)<0 .
\end{aligned}
$$

From (46)-(49) we conclude that at time $T-1$ control law 1 is optimal for $\lambda+\mu<1$ and control law 2 is optimal for $\lambda+\mu>1$.

When $\lambda<\frac{1}{2}<\mu$ we have been unable to characterize optimal routing strategies. It is worthwhile investigating whether there exists a routing strategy that is optimal for all $\lambda, \mu$ such that $\lambda+\mu<1(\lambda+\mu>1)$.

It is interesting to note that even for this simple problem the optimal routing strategies depend on the values of the service and arrival rates. This feature of 
the optimal solution is in sharp contrast with the results of Ephremides et al. (1980), where the centralized version of our problem is formulated and the optimal routing strategy is expressed by the "join-the-shortest-queue" rule for any values of the arrival and service rates. The characteristics of the optimal decentralized routing strategy is another manifestation of the subtleties arising in decentralized routing problems. Since in our problem information is decentralized and there are two controllers instead of one, the optimal routing strategy is characterized by the rule "keep-the-queue-lengths-as-balanced-as-possible." Consequently, the controllers have to coordinate their actions to satisfy the above rule. Coordination depends on the controllers' perception of the likelihood of the queue length that they don't observe. This likelihood depends on the arrival and service rates. Therefore, different arrival and service rates require different optimal coordination and hence different optimal routing decisions. The description of the optimal strategy given above and the detailed analysis presented in Pandelis and Teneketzis (1993) show that optimal coordination between the two controllers is a subtle issue.

\section{Conclusions}

The problem analyzed in this paper reveals some of the complications and subtleties arising in routing under decentralized information. The symmetry in the system and the information structure results in considerable simplification in the analysis of the problem and leads to an optimal routing strategy that is characterized by the simple rule "keep-the-queue-lengths-as-balanced-as-possible." The challenge is to extend the analysis to deal with decentralized routing problems where some of the symmetry present in this paper is lost.

Acknowledgements: The authors thank the anonymous reviewer whose comments helped improve the presentation of the paper. This research was supported in part by NSF Grant NCR-9204419 and by the University of Michigan ITS Research Center of Excellence Grant No. 304025.

\section{References}

[1] Bell CW, Stidham S (1983) Individual versus social optimization in the allocation of customers to alternative servers. Management Sci 29:831-839

[2] Beutler F, Teneketzis D (1989) Routing in queueing networks under imperfect information: Stochastic dominance and thresholds. Stoch Stoch Rep 26:81-100 
[3] Boel RK, van Schuppen JH (1989) Distributed routing for load balancing. Proc IEEE, Special Issue on Dynamics of Discrete Events Systems 210-221

[4] Chang C-S (1992) A new ordering for stochastic majorization: Theory and applications. Adv Appl Prob 24:604-634

[5] Davis E (1977) Optimal control of arrivals to a two-server queueing system with separate queues. PhD dissertation, Program in Operations Research, North Carolina State University, Raleigh, NC

[6] Ephremides A, Varaiya P, Walrand J (1980) A simple dynamic routing problem. IEEE Trans Automat Control 25:690-693

[7] Hajek B (1984) Optimal control of two interacting service stations. IEEE Trans Automat Control 29:491-499

[8] Hajek B (1990) Performance of global load balancing by local adjustment. IEEE Trans Inform Theory $36: 1398-1414$

[9] Hordijk A, Koole G (1990) On the optimality of the generalized shortest queue policy. Prob Eng Inf Sci 4:477-489

[10] Lin W, Kumar PR (1984) Optimal control of a queueing system with two heterogeneous servers. IEEE Trans Automat Control 29:696-703

[11] Pandelis DG, Teneketzis D (1993) A simple load balancing problem with decentralized information. Control Group Report No CGR-93-13, Department of Electrical Engineering and Computer Science, University of Michigan, Ann Arbor, MI

[12] Ross S (1983) Stochastic processes. Wiley, New York

[13] Stamoulis GD, Tsitsiklis JN (1991) Optimal distributed policies for choosing among multiple servers. Proc 30th CDC, Brighton, UK 815-820

[14] Stidham S (1985) Optimal control of admission to a queueing system. IEEE Trans Automat Control 30:705-713

[15] Varaiya P, Walrand J (1978) On delayed sharing patterns. IEEE Trans Automat Control 23:443-445

[16] Walrand J (1984) A note on "Optimal control of a queueing system with two heterogeneous servers". System Control Lett 4:131-134

[17] Weber RR (1978) On the optimal assignment of customers to parallel servers. J Appl Prob $15: 406-413$

[18] Winston W (1977) Optimality of the shortest line discipline. J Appl Prob 14:181-189

[19] Witsenhausen HS (1971) Separation of estimation and control for discrete time systems. Proc IEEE 59:1559-1566

[20] Whitt W (1986) Deciding which queue to join: Some counterexamples. Oper Res 34:55-62

[21] Xu SH (1992) Socially and individually optimal routing of stochastic jobs in parallel processor systems. Oper Res 40:367-375

Received: January 1995

Revised version received: May 1995 